\title{
New Approach to Simulation of Heat State of Compartments from Lattice Composite Shells for Space Engineering Products
}

\author{
Alexander F. Razin ${ }^{1}$, Mikhail N. Slitkov, ${ }^{1}$ and Anatoly N. Garashchenko, ${ }^{1, *}$ \\ ${ }^{1}$ Central Research Institute for Special Machinery, JSC, Khotkovo, Moscow Region, Russia
}

\begin{abstract}
A new approach to the simulation of the heat state of the compartment of lattice polymer composite materials (PCM), not providing for the use of known commercial software packages, has been proposed. The simulation has been performed using the PCM interstage of the Proton rocket as an example with due account of aerodynamic heating, solar radiation and acting of jets of auxiliary propulsion units. At the first stage of numerical analysis, a problem of unsteady heat conduction in the system "skin-air gap-heat insulation" has been solved. An effect of changing a pressure inside a compartment on thermal conductivity of heat insulation was taken into account. The effective thermal conductivity in gaps was used. An effect of a temperature of equipment on a value of radiant heat flux was also taken into account. At the second stage, the heat state of the system "skin-rib" was analyzed. A mathematical model in the form of a system of nonlinear equations for heat balance of control elements on which a rib and a skin section were partitioned, including an information about a temperature of heat insulation received at the first stage of the simulation, was used.
\end{abstract}

\section{Introduction}

The creation of up-to-date rocket and space technology provides for an analysis of temperature fields of both separated structural elements and a product as a whole in connection with high level and variety of heat loads on majority of units and their blocks. The determination of temperature fields (or even if characteristic temperatures of separate sections of the most heated surfaces) under actual operational conditions is practically impossible.

Therefore, studies in this field show that the mathematical simulation is the main instrument for that analysis. At the same time, the simulation of heat transfer processes proceeding in complex, as a rule multiply connected geometrically and physically, fields of products of rocket and space technology is an independent problem difficult to solve.

\footnotetext{
* Corresponding author: a.n.gar@mail.ru
} 
Known methods and approaches do not provide the possibility of its solution. Main difficulties of numerical solution of heat transfer problems for structures, for example, of the PCM lattice compartment type (Fig. 1) lie in substantial non-one-dimensionality of heat transfer conditions, non-uniformity of heat fluxes on boundaries of a solution range (outer and inner contours of a product), multiple lamination of actual structures, breaks of transport factors on these boundaries, substantial contribution of radiation and convection to forming the temperature fields of a specific object.
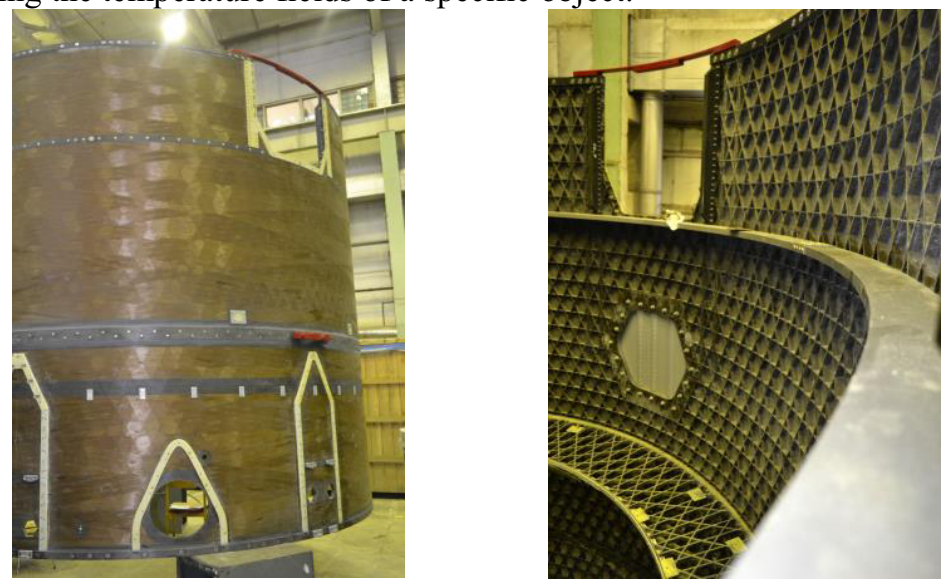

Fig. 1. Appearance of the compartment from the outside and on the inside.

To solve this kind of nonlinear boundary problems of mathematical physics, not only numerical methods [1] but and specific methodical and algorithmic techniques [2-5] of obtaining reliable theoretic consequences, for example, when studying natural convection processes in areas with local power sources [2], igniting of structurally non-uniform substances [3], natural convection in closed areas with radiant heat sources [4], igniting of fuels under local heating [5] have been developed. Solutions of singular-disturbed problems [2-5] are obtained by finite difference method for irregular and non-uniform, basically, difference nets. With this, to overcome one or several above-listed problems, the use of very small steps of a difference net in time (up to $10^{-7} \mathrm{~s}$ ) was the most reliable technique. However, using this efficient, but taking long time for calculations, technique when solving heat transfer problems for products of rocket and space technology is impossible in connection with their large sizes and complex configuration, high heat loads and long operation time. These reasons caused the objective necessity of developing a new approach to the simulation of heat transfer processes in large-sized and non-uniform property materials of separate elements of structures for long time, various heat loading conditions and high heat fluxes to heating surfaces.

The objective of the present paper is to analyze unsteady temperature fields in PCM structural elements made in the form of carbon reinforced plastic lattice structures that are distinguished by high load-bearing capacity at minimum mass $[6,7]$. Lately, such structures began to be widely used as rocket compartments in space technology. They are applied, for example, when making the interstage (upper) and the skirt of the tail compartment for the stage II of the Proton rocket, compartments for the Angara rocket, etc.

\section{Statement of the problem}

The new approach was used to the mathematical simulation of heat transfer in PCM lattice structures with complex configuration. Heating of the outer side of a compartment at the 
expense of convection and heat transfer on inner surfaces from equipment located in an object at the expense of radiation and natural convection were considered. Besides, at the end of the system work, the action of jets of auxiliary propulsion units (PU) on the compartment surface was taken into account.

The mathematical simulation was carried out for a compartment in the form of a lattice shell (outside diameter of $4100 \mathrm{~mm}$, length of $3731 \mathrm{~mm}$ ) formed by a set of spiral and annular ribs, with a skin. Its appearance is shown in Figure 1 and the layout of equipment blocks (II \& III) is presented in Fig. 2. The thickness of a skin was $1.5 \mathrm{~mm}$, of a spiral rib was $6 \mathrm{~mm}$ and of an annular rib was $4 \mathrm{~mm}$. The inner surface of the compartment is covered by heat-insulating material. There is a gap between the material and the skin equal to the height of ribs $(23 \mathrm{~mm})$. The skin consists of layers of different PCMs and outer heatprotective coating (OHPC) whose thickness depends on the skin section. The outer surface (OHPC surface) is covered with white enamel. It is subject to aerodynamic heating.

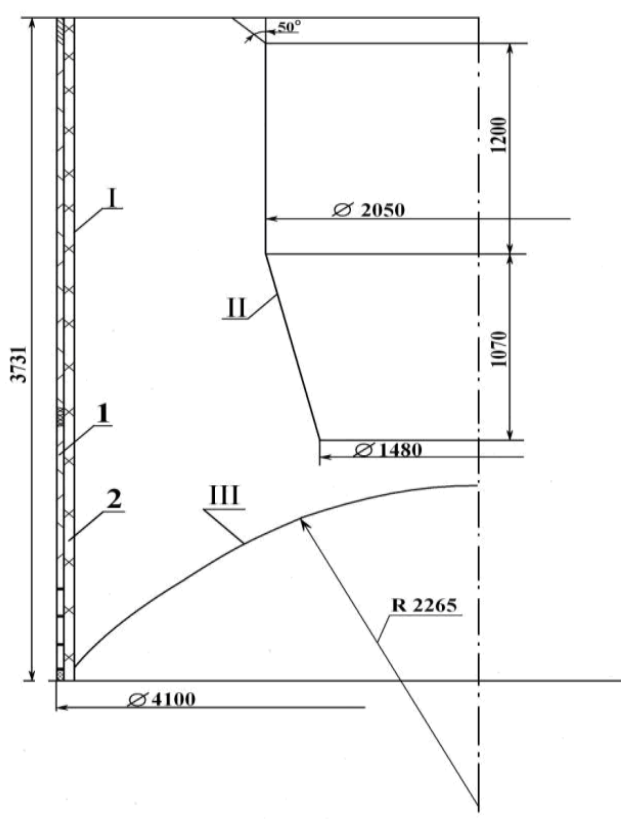

Fig. 2. Layout of equipment in inner volume of the compartment: 1 - shell; 2 - heat insulation; II, III - equipment blocks.

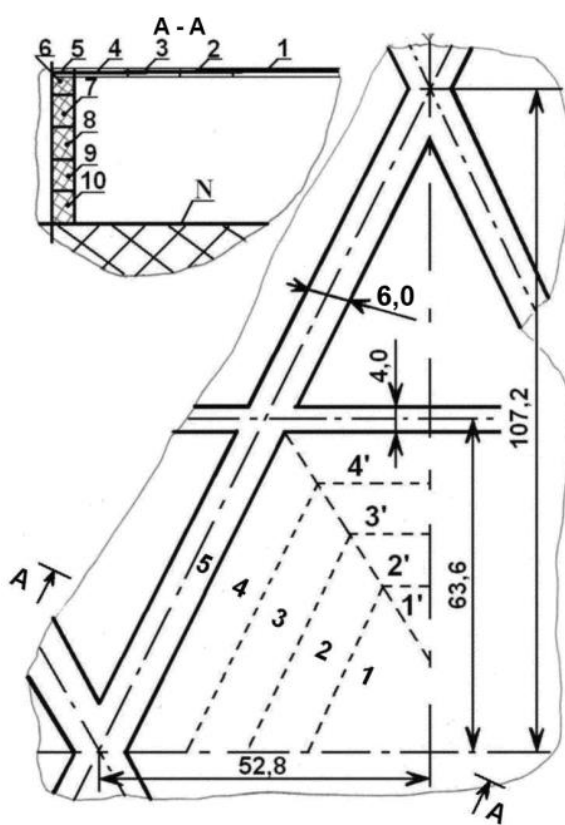

Fig. 3. Figure 3. Diagram of solution area for a skin and ribs $(1,2, \ldots, 10$ are element numbers).

The ambient temperature $T_{\mathrm{e}}(t)$ and coefficient of convective heat exchange $\alpha_{\mathrm{e}}(t)$ are known. From a certain moment of time, the heat flux density $q_{\mathrm{e}}(t)$ on this boundary is given. In addition, at the end of work, the outer surface is subject by the action of jets of auxiliary propulsion units.

At the first stage of simulating, the heat state of the most warmed-up element of the compartment, i.e. a skin, was determined. The mathematical model of the process is a system of nonlinear equations of unsteady heat conduction in multilayered cylinder (skinair gap-heat insulation). It includes boundary conditions on the inner surface that take into account convective heat exchange (up to a moment of pressure drop down to zero) and resulting radiant heat flux. The latter was calculated by method of "radiant balance" in a closed system of bodies [8]. The heat conduction equation to be used has the form: 


$$
c_{\mathrm{m}} \rho_{\mathrm{m}} \frac{\partial T}{\partial t}=\frac{1}{r} \frac{\partial}{\partial r}\left(\lambda_{\mathrm{m}} r \frac{\partial T}{\partial r}\right), \quad t>0, r_{0}<r<r_{\mathrm{n}}, \quad r_{\mathrm{m}}=r_{0}+\sum_{\mathrm{m}=1}^{\mathrm{m}} \delta_{\mathrm{m}}, \quad m=1,2 \ldots, n,
$$

where $T, r, t$ are temperature, radial coordinate and time;

$c, \rho, \lambda$ are specific heat capacity, density and thermal conductivity;

$m$ is number of a layer of multilayered cylinder.

This model has features consisting in the account of an influence of the pressure change inside a compartment on thermal conductivity of heat insulation and the use of effective thermal conductivity in a gap between a skin and heat insulation with due account of reemission. The natural-convective heat transfer coefficient and the air temperature in inner volume of a compartment were parameters of the mathematical model for calculating of which the nonlinear differential heat-balance equation was used.

The OHPC thicknesses were selected so that the skin temperature during the all process of heating (including the action of propulsion unit jets) did not exceed the level of beginning thermal destruction of a binder $\left(\sim 210^{\circ} \mathrm{C} \ldots 220^{\circ} \mathrm{C}\right)$. In places of affecting PU, values of OHPC thicknesses were determined with due account of removal values $\left(\delta_{d}\right)$ which were estimated from the equation:

$$
\delta_{\mathrm{d}}=\frac{Q}{\rho H_{\mathrm{ef}}}=\frac{1}{\rho H_{\mathrm{ef}}} \int_{0}^{t^{*}} \alpha\left(T_{\mathrm{e}}-T_{\mathrm{d}}\right) d t,
$$

where $Q$ is an integral of heat flux density;

$H_{\mathrm{ef}} \& T_{\mathrm{d}}$ are effective enthalpy and OHPC destruction temperature.

\section{Results and discussion}

A high number of estimation values of the OHPC thickness as well as non-uniformity of heat flux to the outer surface during the work caused the necessary to analyze the heat state of a skin on many its sections. The results of simulating the temperature fields of different sections are obtained in the form of time variation of temperatures on boundaries of skin material layers and heat insulation surface. As an example, Fig. 4 presents temperature values for one of the sections.

Temperature fields of a skin were used at the second stage of simulating when analyzing the heat state of spiral and annular ribs of a compartment. For this, a mathematical model in the form of a system of nonlinear equations of heat balance for control volumes of elements into which a rib and a skin section being adjacent to it and dissected by adiabatic planes as is shown in Fig. 2 were partitioned. With such partitioning, it was adopted that a temperature in all volume of an element is the same (temperature field is uniform). When stating a problem, it is assumed that outer surfaces of elements (from 1 to 5) are affected by heat flux analogous to one to be taken into account before on the outer surface of a skin. With this, the skin sections whose temperatures are the most high and were calculated formerly (see Fig. 4) were considered. Each of the skin elements under consideration (besides an element 5) participates in the process of radiant heat exchange with elements of a rib $(6 \ldots 10)$ and the heat-insulating material surface $(N)$ whose temperature is adopted known from the results of calculations conducted before. 


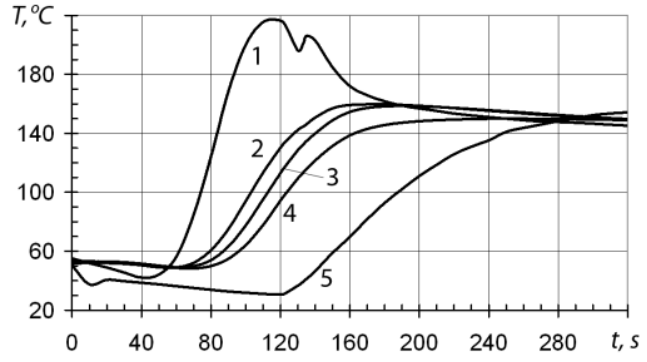

Fig. 4. Temperature changes in elements of system "skin - air gap - heat insulation": 1- OHPC outer surface; 2 - OHPC-skin boundary; 3 - inner surface of a skin; 4 - outer surface of heat insulation; 5 - inner surface of heat insulation.

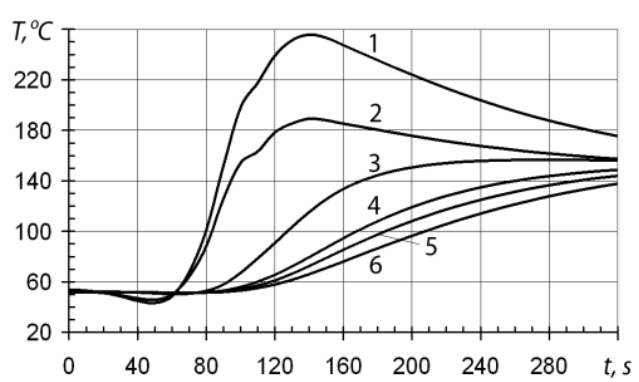

Fig. 5. Temperature changes in annular rib with skin sections being adjacent to it: 1 skin element (No. 1); 2 - skin element at a rib (No. 5); 3, 4, 5 - rib elements Nos. 8, 9, 10 (respectively).

It is also assumed that the heat transfer from element to element is carried out only by heat conduction. The heat exchange between elements within mutual "visibility" occurs at the expense of radiation. A system of equations is stated for skin sections as well as for sections on a rib. For sections of a skin between ribs, this system has the following form:

$$
C_{\mathrm{i}} \frac{d T_{\mathrm{i}}}{d t}=q_{\mathrm{i}} F_{\mathrm{i}}-\sum_{\mathrm{j}=\mathrm{m}+1}^{\mathrm{j}=\mathrm{n}} K_{\mathrm{i}-\mathrm{j}}\left(T_{\mathrm{i}}-T_{\mathrm{j}}\right)-K_{\mathrm{i}-\mathrm{j}+1}\left(T_{\mathrm{i}}-T_{\mathrm{j}}\right)-K_{\mathrm{i}-\mathrm{N}}\left(T_{\mathrm{i}}-T_{\mathrm{N}}\right) .
$$

For section of a skin on a rib:

$$
C_{\mathrm{m}} \frac{d T_{\mathrm{m}}}{d t}=q_{\mathrm{m}} F_{\mathrm{m}}-K_{\mathrm{m}-\mathrm{j}}\left(T_{\mathrm{m}}-T_{\mathrm{j}}\right) .
$$

For sections of a rib:

$$
C_{\mathrm{j}} \frac{d T_{\mathrm{j}}}{d t}=q_{\mathrm{i}} F_{\mathrm{i}}-\sum_{\mathrm{j}=\mathrm{m}+1}^{\mathrm{j}=\mathrm{n}} K_{\mathrm{i}-\mathrm{j}}\left(T_{\mathrm{i}}-T_{\mathrm{j}}\right)-K_{\mathrm{j}-\mathrm{j}+1}\left(T_{\mathrm{j}}-T_{\mathrm{j}+1}\right)-K_{\mathrm{j}-\mathrm{N}}\left(T_{\mathrm{j}}-T_{\mathrm{N}}\right) .
$$

The following symbols have been adopted: $C$ is heat capacity of element to be determined as $C=\rho c V$ ( $V$ is element volume); $F$ is surface area of element; $\varepsilon$ is emissive power; indices: $i=1, \ldots, m$ is symbol of element on a skin, $j=m+1, \ldots, n$ is symbol of element on a rib (in this case $n=5, m=5$ ); $e$ is environment; $w$ is outer surface of a skin; $N$ is heat-insulation surface.

Density of the resulting heat flux on the outer surface of skin elements as well as radiant heat transfer from $i$-th skin section to $j$-th rib section are calculated from the formulae [8]:

$$
\begin{aligned}
& q_{\mathrm{i}}=\alpha_{\mathrm{e}}\left(T_{\mathrm{e}}-T_{\mathrm{i}}\right)+q_{\mathrm{e}}-\varepsilon_{\mathrm{N}} \sigma T_{\mathrm{i}}^{4} \\
& K_{i-j}=F_{i-j} \sigma \varepsilon_{\mathrm{i}} \varepsilon_{\mathrm{j}} \varphi_{\mathrm{i}-\mathrm{j}} \frac{T_{\mathrm{i}}^{4}+T_{\mathrm{j}}^{4}}{T_{\mathrm{i}}-T_{\mathrm{j}}},
\end{aligned}
$$

where $K_{\mathrm{i}-\mathrm{j}}$ is parameter of heat transfer by effective heat conduction from heater to wall, 
taking into account re-emission of surfaces and natural convection in plane slit [8];

$F_{\mathrm{i}-\mathrm{j}}$ is area of mutual irradiation of two elements;

$\varphi_{\mathrm{i}-\mathrm{j}}$ is angular coefficient.

Typical results of temperature computations for the annular rib (which is more warmed up with comparison to the spiral rib) with skin sections being adjacent to it are presented in Fig. 5 in the form of temperature-time relations for each element. In this case, a crosssection with formerly determined temperature of a skin was considered (see Fig. 4). From results of simulating it follows, in particular, that maximum temperature for a rib $\left(139{ }^{\circ} \mathrm{C}\right)$ is reached by the end of heat effect.

The truth of numerical temperature values was verified by comparison for the heat state of a skin obtained by traditional and above-described methods for element under No. 1 (see Fig. 3) which is subject to the influence of ribs to a least extent.

The similar approach was applied when simulating the time variation of temperatures of elements in the tail compartment of the Proton rocket. The load-bearing structure of its compartment case consists of similar lattice shell formed by a family of spiral and annular ribs and a skin. The skirt case has a cylindrical shape $2390 \mathrm{~mm}$ in length and an outside diameter of $4100 \mathrm{~mm}$.

For the interstage and tail compartments of the rocket, similar simulating was the important part of their design and development. The serviceability of the compartments has been confirmed by the results of flight tests. Besides, the considered method was successfully used while simulating the heat state of the Angara rocket compartments.

\section{Conclusion}

A new approach to the simulation of the heat state of structural elements of a polymer composite lattice compartment has been proposed. This approach was used when designing and developing of various products with a complex configuration under conditions of an intensive heat effect. The fidelity of calculations has been confirmed by the results of flights of the rockets with compartments under consideration.

\section{References}

1. V.L. Strakhov, A.N. Garashchenko, G.V. Kuznetsov, V.P. Rudzinskii, Combust. Explos. Shock Waves 37, 2 (2001)

2. G.V. Kuznetsov, M.A. Sheremet, Int. J. Heat Mass Tran. 54, 260 (2011)

3. O.V. Vysokomornaya, G.V. Kuznetsov, P.A. Strizhak, Russ. J. Phys. Chem. B 5, 4 (2011)

4. G.V. Kuznetsov, N.I. Kurilenko, V.I. Maksimov, G.Ya. Mamontov, T.A. Nagornova, J. Eng. Phys. Thermophys. 86, 3 (2013)

5. D.O. Glushkov, G.V. Kuznetsov, P.A. Strizhak, Russ. J. Phys. Chem. B, 5, 6 (2011)

6. V.V. Vasiliev, A.F. Razin, Compos. Struct. 40, 1-2 (2006)

7. V.V. Vasiliev, V.A. Barynin, A.F. Razin, Compos. Struct. 94, 3 (2012)

8. E.M. Sparrow, R.D. Cess, Radiation Heat Transfer (Hemispere Publishing Corporation, USA, 1978) 